\title{
The Beetle (Coleoptera) and True bug (Heteroptera) species pool of the alpine "Pian di Gembro" wetland (Villa di Tirano, Italy) and its conservation
}

\begin{abstract}
The Coleoptera and Heteroptera species pool was investigated in the "Pian di Gembro" wetland (Villa di Tirano, Sondrio, Italy). The wetland consists of a bog and its surroundings, referred to as wetland components, that are both subjected to a diversified intermediate management regime (DIMR). The application of the DIMR for plant species conservation resulted in the establishment of 11 wetland zones with a characteristic vegetation. In a three year sampling program, 997 Coleoptera and Heteroptera representing 141 species from 14 families were collected. Among these species, 64 species share both wetland components, 11 are restricted to the bog and 63 were found in the surroundings only. Among the species pool there were 23 tyrphophile taxa and only one tyrphobiont. With the exception of one zone, all zones are inhabited by zone-specific species. By taking into account both the general species pool and the pool of species of particular interest to conservationists, only one zone can be considered as redundant since it is inhabited by species that occur also in other zones. Hence, all the zones, with one exception, are effective for species pool conservation. The existing DIMR implemented for plant species conservation is also effective for conserving the species pool of Coleoptera and Heteroptera.
\end{abstract}

Riassunto - Nel presente studio sono state indagate le specie di Coleotteri ed Eterotteri presenti nell'area umida di Pian di Gembro (Villa di Tirano, Sondrio, Italia), costituita da una componente di torbiera e da ambienti ecotonali circostanti sottoposti entrambi ad un regime di gestione intermedia diversificata (DIMR). L'applicazione di strategie DIMR nella gestione e conservazione delle specie vegetali ha portato alla determinazione di 11 zone umide con vegetazione caratteristica, all'interno delle quali si sono svolti i campionamenti. Il programma di campionamento, della durata di tre anni, ha permesso di raccogliere 997 campioni di Coleotteri ed Eterotteri appartenenti a 141 specie e 14 famiglie. Tra queste specie, 64 sono state censite in entrambe le componenti della zona umida, 11 sono ristrette alla torbiera e 63 agli ecotoni circostanti. Nel gruppo di specie censite sono presenti 23 taxa tirfofili e uno solo tirfobionte. Tutte le stazioni di raccolta, eccetto una, presentano specie uniche. Considerando quelle di interesse conservazionistico, solo una zona può dirsi ridondante, in quanto le specie censitevi sono presenti in altre zone. Concludendo, 10 delle 11 zone che compongono la zona umida di Pian di Gembro sono utili nella 
conservazione dello "species pool". La strategia di gestione DIMR, utilizzata per la conservazione delle specie vegetali risulta essere funzionale alla conservazione delle specie di Coleotteri ed Eterotteri presenti.

Key words - bog, insect coenoses, species pool, conservation categories.

\section{INTRODUCTION}

Wetlands are ecologically sensitive adaptive systems and much attention has been given to the design and implementation of adequate management strategies (Turner et al.2000). Because of their capacity to conserve species of conservation interest and to provide ecosystem services, wetlands are considered as natural capital and often assigned protected status (Spitzer \& Danks, 2006; Fisher et al., 2009).

The term "protected area" refers to any area of land or sea managed for the persistence of biodiversity and other natural processes, achieved through constraints on incompatible land uses (Possingham et al., 2006). Despite high levels of protection and adequate management within their borders, many protected areas are not functioning as originally envisioned. Agriculture, settlements, and other human land uses in the unprotected part of the ecosystem, as well as the lack of any management, may alter the flow of energy, material and organisms across the ecosystems in ways that change ecological functioning within protected areas (Hansen \& DeFries, 2007). Wetlands are areas whose soil is saturated with moisture either permanently or seasonally. Nevertheless, the importance of the surroundings for the integrity of the bog and the interest in conservation measures motivated us to apply the term 'wetland' to a bog and its surroundings and refer to them as 'wetland components'.

In the wetland under study, both the bog and its surroundings are subjected to diversified management procedures whose frequency and intensity change through time in different zones. Importantly, the diversification of these practices falls into range that maintains the integrity of the wetland and prevents it from shifting into an irreversible late successional state where the desirable wetland characteristics are lost (Andreis \& Rodondi, 1982). Ecological theory predicts that species diversity is highest under intermediate disturbance (Smith \& Smith, 2001). A combination of diversified and intermediate management regimes (DIMR) holds to promise to conserve both the species pool and a high species diversity (Guo, 2003). Specific adaptations may restrict species to either the bog or its surroundings or limit their distribution to particular zones resulting from the application of the DIMR to the two wetland components. While detailed information is available for northern wetlands (Spitzer \& Danks, 2006), little is known on the diversity and the pool of species inhabiting Alpine wetlands. The limited information is restricted to specific taxa such as Odonata (Marcuzzi, 1948; Balestrazzi et al. 1983), Heteroptera (Rampazzi \& Dethier, 1997; Montagna et al., 2008), Coleoptera (Focarile, 1957) and Trichoptera (Cianficconi et al., 2005).

A DIMR is applied for plant species conservation to the 'Pian di Gembro' wetland in the northern Italian Alps (Andreis \& Rodondi, 1982; Andreis \& Rodondi, 2005). 
The application of the DIMR produced 11 different ecological zones corresponding to habitat typologies (Carta Habitat Natura 2000 IT2040025). This work deals with Beetles (Coleopera) and True bugs (Heteroptera) as important elements of the insect species pool.

\section{MATERIAL AND METHODS}

\section{Study site}

The 'Pian di Gembro' wetland is located north of the Aprica Pass at $1350 \mathrm{~m}$ above sea level in Villa di Tirano, Sondrio (Italy) (Fig. 1). With other alpine wetlands, it shares the features of raised and blanket bogs characterized by a mosaic of plant associations (Andreis \& Rodondi, 1982). Since 1988, the wetland and the surrounding area has become a Regional Reserve and Site of Community Importance (SCI IT2040025) under the Habitat Directive Act (92/43/EEC). The protected area covers 126.5 ha, and the major and minor diameters measure $2000 \mathrm{~m}$ and $300 \mathrm{~m}$, respectively. Coniferous forests, deciduous trees and shrubs, as well as pastures and meadows, surround the bog. Though no synthetic fertilizers are applied in the wetland, it receives nitrogen and sulphur from the atmosphere (Krupa, 2003; Erisman et al., 2005).

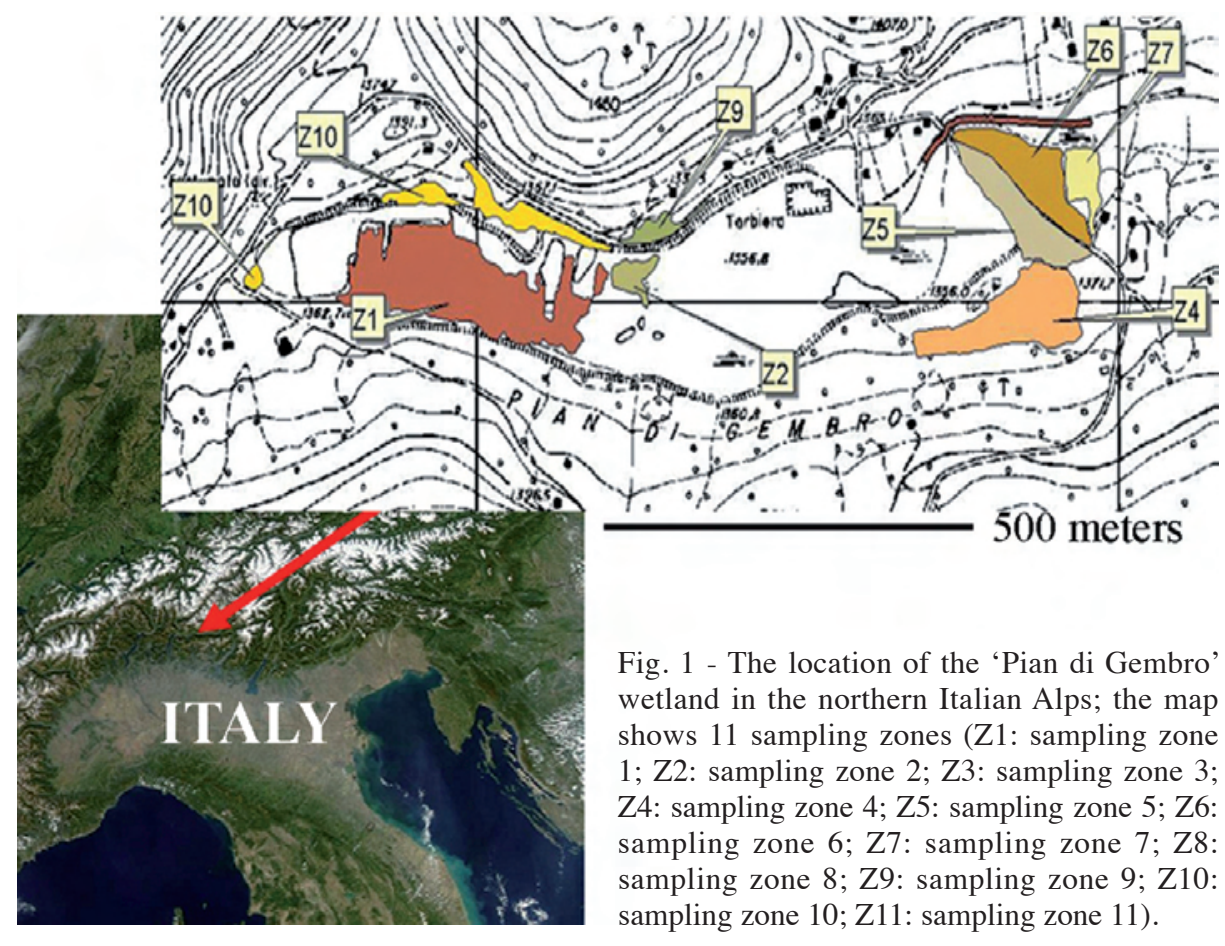




\section{Sampling}

The sampling program started in 2005 and continued until 2007. It was restricted to the snow-free periods lasting from March to October. Sampling was carried out in the different zones corresponding to the habitat typologies specified in the Habitat Directive 92/43/EEC part. I and identified by a numeric code. For the protected wetland, the Carta Habitat Natura 2000 IT2040025 establishes habitat typologies and allows the identification of 11 zones (Table 1).

During 2005, one standard pitfall trap (Mason et al., 2002; Liu et al., 2007; Uys et al., 2010) was deployed in each zone (Table 1); to improve the sampling coverage, an additional trap was put into each of the larger sampling zones 1,2 and 4. The pitfall traps were baited with different types of attractants such as meat, fish, beer, banana and a water solution of $\mathrm{NaCl}$ (Mason et al., 2002). A total of 19 traps were deployed in the wetland and visited every 10 days for 7 months per year. To improve the sampling efficiency, the pitfall trap technique was complemented by other sampling techniques including the use of sweep nets and entomological umbrellas (Mason et al., 2002), sieves, and direct observations of specimens in specific micro-habitats (e.g. tree trunks, underneath stones, and at the bottom of Carex spp.).

Table 1 - The sampling zones located within the "Pian di Gembro" wetland components, the description of the vegetation and the habitat code (Carta Habitat Natura 2000 IT2040025) (Z1: sampling zone 1; Z2: sampling zone 2; Z3: sampling zone 3; Z4: sampling zone 4; Z5: sampling zone 5; Z6: sampling zone 6; Z7: sampling zone 7; Z8: sampling zone 8; Z9: sampling zone 9; Z10: sampling zone 10; Z11: sampling zone 11).

\begin{tabular}{|c|c|c|c|}
\hline $\begin{array}{l}\text { Wetland } \\
\text { components }\end{array}$ & Zones & Description of the vegetation & Habitat code \\
\hline \multirow[t]{4}{*}{ Bog } & $\mathrm{Z} 1$ & Pools with Utricularia spp. community & 3160 \\
\hline & $\mathrm{Z} 2$ & $\begin{array}{l}\text { Patch vegetation with Trichophorum spp. and } \\
\text { Molinia spp. }\end{array}$ & 7140 and 7150 \\
\hline & $\mathrm{Z3}$ & Carex lasiocarpae community & 7140 \\
\hline & $\mathrm{Z4}$ & Trichophorum caespitosum community & 7140 \\
\hline \multirow[t]{3}{*}{ Surroundings } & $\mathrm{Z} 5$ & Calluna dry heaths & 4030 \\
\hline & $\mathrm{Z6}$ & Calluna dry heaths & 4030 \\
\hline & $\mathrm{Z7}$ & Mountain hay meadows & 6520 \\
\hline $\begin{array}{l}\text { Drainage } \\
\text { channel }\end{array}$ & Z8 & Drainage channel vegetation & \\
\hline \multirow[t]{3}{*}{ Surroundings } & Z9 & Managed Calluna dry heaths & 4030 \\
\hline & $\mathrm{Z} 10$ & Calluna dry heaths & 4030 \\
\hline & $\mathrm{Z} 11$ & Mountain hay meadows & 6520 \\
\hline
\end{tabular}




\section{Species pool and species of particular conservation interest}

The collected specimens were brought to the laboratory for identification at the species level. The individuals were assigned to different families and sent to specialists who identified the species.

First, the insects were grouped into three main coenoses: $i$ ) species occurring in the bog, ii) species inhabiting the surroundings, and iii) species distributed over both the bog and the surroundings. Thereafter, species and families were listed according to the different zones in which they were captured. Subsequently, the species specific to a zone were separated from the ones found in more than one zone. Second, we assessed the species pool by selecting species of particular interest to conservationists. The scientific literature and experts were consulted to establish the categories of interest: a) species of humid biotopes (tyrphophiles); b) bioindicator species; c) endemic alpine taxa; d) species with boreo-alpine distribution; e) species of mountainous environments; f) species of lowland environments; g) species with wide ecological tolerance to the effect of abiotic factors.

\section{RESULTS}

\section{Species pool}

The sampling program made available 997 individuals representing 141 species from 11 families of Heteroptera (Anthocoridae, Nabidae, Miridae, Reduviidae, Coreidae, Rhopalidae, Lygaeidae, Acanthosomatidae, Pentatomidae, Scutellaridae, Thyreocoridae) and three families of Coleoptera (Carabidae, Chrysomelidae sensu latu, Curculionidae sensu latu). Table 2 lists the 141 collected species, the number of specimens and the sampling zones in which the specimens were collected.

\section{Species grouping}

The insects listed in Table 2 belong to three main coenoses. The first coenosis is composed of species exclusively found in the bog (zones 1,2 or 3, Table 3 ) and consists of 11 species including 5 species of Heteroptera, 2 species of Carabidae, 3 species of Chrysomelidae and 1 species of Curculionidae. Of note, the tyrphophiles Donacia obscura, Limnobaris dolorosa and Platysma oenotrium are members of this coenosis. The second coenosis consists of 63 species exclusively found in the surroundings (zones $5,6,7,8,9$ or 10 , Table 3 ). These insects belong to 21 species of Heteroptera, 9 species of Carabidae, 21 of Chrysomelidae and 12 of Curculionidae. The most interesting species of this coenosis are the four endemic alpine taxa Cryptocephalus sericeus sp. zambanellus, Otiorhynchus scaber, O. frigidus and Pterostichus dissimilis that are of conservation interest (Osella et al., 2005). The third coenosis is composed of 64 species that are found in both the bog and the surroundings (Table 4).

The highest number of species specific to one zone were found in zones 10 (23 
Table 2 - Species list of Beetles (Coleoptera) and True bugs (Heteroptera) sampled in the "Pian di Gembro" wetland. The number of indiviaduls is given for each sampling zone (Z1: sampling zone 1; Z2: sampling zone 2; Z3: sampling zone 3; Z4: sampling zone 4; Z5: sampling zone 5; Z6: sampling zone 6; Z7: sampling zone 7; Z8: sampling zone 8; Z9: sampling zone 9; Z10: sampling zone 10; Z11: sampling zone 11).

\begin{tabular}{|c|c|c|c|c|c|c|c|c|c|c|c|}
\hline Species & $\mathbf{Z 1}$ & $\mathbf{Z 2}$ & $\mathbf{Z 3}$ & $\mathbf{Z 4}$ & $\mathbf{Z 5}$ & $\mathbf{Z 6}$ & $\mathbf{Z 7}$ & $\mathbf{Z 8}$ & $\mathbf{Z 9}$ & Z10 & Z11 \\
\hline Temnostethus pusillus (Herrich-Schäffer 1835) & 0 & 0 & 0 & 0 & 1 & 0 & 0 & 0 & 0 & 0 & 0 \\
\hline Tetraphleps bicuspis (Herrich-Schäffer 1835) & 2 & 0 & 0 & 0 & 0 & 0 & 1 & 0 & 0 & 0 & 0 \\
\hline Nabis flavomarginatus Scholz 1847 & 0 & 0 & 0 & 0 & 0 & 0 & 0 & 0 & 0 & 0 & 1 \\
\hline Nabis rugosus (Linnaeus 1758) & 0 & 0 & 0 & 0 & 4 & 0 & 0 & 0 & 0 & 6 & 0 \\
\hline Alloeotomus gothicus (Fallén 1807) & 0 & 0 & 0 & 0 & 0 & 0 & 1 & 0 & 0 & 0 & 0 \\
\hline Deraeocoris ruber (Linnaeus 1758) & 1 & 0 & 0 & 0 & 0 & 0 & 0 & 0 & 0 & 1 & 0 \\
\hline Halticus apterus (Linnaeus 1761) & 1 & 0 & 0 & 0 & 0 & 0 & 1 & 0 & 0 & 2 & 0 \\
\hline Adelphocoris seticornis (Fabricius 1775) & 0 & 0 & 0 & 0 & 1 & 0 & 1 & 0 & 0 & 0 & 0 \\
\hline Camptozygum aequale (Villers 1789) & 2 & 0 & 0 & 0 & 0 & 0 & 0 & 0 & 0 & 0 & 0 \\
\hline Capsus ater (Linnaeus 1758) & 2 & 0 & 0 & 0 & 2 & 0 & 0 & 1 & 0 & 3 & 0 \\
\hline Charagochilus gyllenhalii (Fallén 1807) & 0 & 0 & 0 & 0 & 0 & 0 & 0 & 0 & 0 & 1 & 0 \\
\hline Leptopterna dolabrata (Linnaeus 1758) & 0 & 0 & 0 & 0 & 2 & 0 & 1 & 0 & 2 & 2 & 2 \\
\hline Lygus pratensis (Linnaeus 17 & 0 & 0 & 0 & 0 & 1 & 6 & 0 & 0 & 0 & 3 & 0 \\
\hline Lygus wagneri Remane 1955 & 0 & 0 & 0 & 0 & 1 & 0 & 0 & 0 & 0 & 1 & 0 \\
\hline Megaloceroea recticornis (Geoffroy 1785) & 0 & 0 & 0 & 0 & 0 & 0 & 0 & 0 & 1 & 1 & 0 \\
\hline Notostira elongata (Geoffroy 1785) & 0 & 0 & 0 & 0 & 0 & 0 & 1 & 0 & 0 & 0 & 0 \\
\hline Polymerus palustris (Reuter 1905) & 0 & 0 & 0 & 0 & 1 & 0 & 0 & 0 & 0 & 3 & 0 \\
\hline Stenodema calcarata (Fallén 1807) & 2 & 2 & 0 & 0 & 0 & 0 & 0 & 0 & 0 & 4 & 0 \\
\hline Stenodema holsata (Fabricius 1787) & 1 & 0 & 0 & 0 & 4 & 0 & 0 & 0 & 0 & 7 & 0 \\
\hline Stenodema laevigata (Linnaeus 1758) & 0 & 0 & 0 & 0 & 0 & 0 & 0 & 0 & 0 & 1 & 0 \\
\hline Heterocordylus genistae (Scopoli 1763) & 0 & 0 & 0 & 0 & 0 & 0 & 0 & 0 & 5 & 0 & 0 \\
\hline Chlamydatus pulicarius (Fallén 1807) & 0 & 0 & 0 & 0 & 2 & 0 & 1 & 0 & 0 & 3 & 0 \\
\hline Plagiognathus arbustorum (Fabricius 1794) & 0 & 0 & 0 & 0 & 0 & 0 & 0 & 0 & 0 & 1 & 0 \\
\hline Plagiognathus chrysanthemi (Wolff 1864) & 0 & 0 & 0 & 0 & 2 & 0 & 0 & 0 & 0 & 0 & 0 \\
\hline Coranus subapterus (De Geer 1773) & 0 & 3 & 0 & 0 & 0 & 0 & 0 & 0 & 0 & 0 & 0 \\
\hline Rhynocoris annulatus (Linnaeus 1758) & 1 & 0 & 0 & 0 & 0 & 0 & 0 & 0 & 0 & 0 & 0 \\
\hline Coreus marginatus (Linnaeus 1758) & 0 & 0 & 0 & 0 & 0 & 1 & 0 & 0 & 0 & 1 & 0 \\
\hline Corizus hyoscyami (Linnaeus 1758) & 0 & 0 & 0 & 0 & 0 & 0 & 0 & 0 & 1 & 0 & 0 \\
\hline Myrmus miriformis (Fallén 1807) & 22 & 22 & 0 & 1 & 6 & 0 & 0 & 2 & 1 & 5 & 0 \\
\hline Rhopalus maculatus (Fieber 1837) & 0 & 1 & 2 & 0 & 3 & 1 & 1 & 0 & 1 & 6 & 0 \\
\hline Rhopalus parumpuncactus Schilling 1829 & 0 & 0 & 0 & 0 & 0 & 0 & 1 & 0 & 0 & 2 & 0 \\
\hline Stictopleurus punctatonervosus (Goeze 1778) & 5 & 3 & 0 & 0 & 9 & 2 & 0 & 0 & 2 & 10 & 0 \\
\hline Kleidocerys resedae (Panzer 1797) & 0 & 0 & 0 & 0 & 0 & 0 & 1 & 0 & 1 & 1 & 0 \\
\hline Nithecus jacobaeae (Schilling 1829) & 2 & 0 & 0 & 0 & 13 & 1 & 2 & 0 & 1 & 8 & 0 \\
\hline Eremocoris plebejus plebejus (Fallén 1807) & 1 & 0 & 0 & 0 & 0 & 0 & 0 & 0 & 0 & 0 & 0 \\
\hline
\end{tabular}




\begin{tabular}{|c|c|c|c|c|c|c|c|c|c|c|c|}
\hline Gastrodes abietum Bergroth 1914 & 0 & 0 & 0 & 0 & 0 & 0 & 3 & 0 & 3 & 0 & 1 \\
\hline Pachybrachius luridus Hahn 1826 & 0 & 0 & 0 & 0 & 0 & 0 & 0 & 0 & 0 & 1 & 0 \\
\hline Peritrechus geniculatus (Hahn 1832) & 1 & 2 & 0 & 0 & 0 & 1 & 0 & 0 & 0 & 0 & 0 \\
\hline Pterotmetus staphyliniformis (Schilling 1829) & 0 & 0 & 0 & 0 & 0 & 0 & 3 & 0 & 0 & 2 & 0 \\
\hline Rhyparochromus pini (Linnaeus 1758) & 2 & 0 & 0 & 0 & 0 & 0 & 1 & 0 & 0 & 0 & 1 \\
\hline Stygnocoris pygmaeus R.F. Sahlberg 1848 & 0 & 0 & 1 & 0 & 0 & 0 & 0 & 0 & 0 & 0 & 0 \\
\hline Stygnocoris sabulosus (Schilling 1829) & 0 & 0 & 0 & 0 & 8 & 0 & 1 & 0 & 1 & 11 & 0 \\
\hline Trapezonotus desertus Seidenstücker 1951 & 0 & 0 & 0 & 0 & 1 & 0 & 0 & 0 & 0 & 0 & 0 \\
\hline Trapsonotus dispar Stål 1872 & 0 & 0 & 0 & 0 & 0 & 0 & 2 & 0 & 0 & 0 & 0 \\
\hline Cyphostethus tristriatus (Fabricius 1787) & 0 & 0 & 0 & 0 & 0 & 0 & 3 & 0 & 0 & 0 & 0 \\
\hline Elasmostethus interstinctus (Linnaeus 1758) & 0 & 0 & 0 & 0 & 1 & 0 & 0 & 0 & 1 & 1 & 0 \\
\hline Elasmucha grisea (Linnaeus 1758) & 0 & 0 & 0 & 0 & 1 & 0 & 0 & 0 & 1 & 0 & 0 \\
\hline Picromerus bidens (Linnaeus 1758) & 0 & 0 & 0 & 0 & 0 & 0 & 0 & 0 & 0 & 1 & 0 \\
\hline Zicrona caerulea (Linnaeus 1758) & 0 & 0 & 0 & 0 & 0 & 0 & 0 & 0 & 0 & 1 & 0 \\
\hline Aelia acuminata (Linnaeus 1758) & 0 & 0 & 0 & 0 & 1 & 0 & 0 & 0 & 3 & 6 & 0 \\
\hline Carpocoris purpureipennis (De Geer 1773) & 0 & 0 & 0 & 0 & 3 & 0 & 0 & 0 & 0 & 4 & 0 \\
\hline Dolycoris baccarum (Linnaeus 1758) & 2 & 0 & 0 & 0 & 2 & 0 & 1 & 0 & 0 & 4 & 0 \\
\hline Eurydema oleracea (Linnaeus 1758) & 0 & 0 & 0 & 0 & 0 & 0 & 0 & 0 & 0 & 5 & 0 \\
\hline Holcostethus vernalis (Wolff 1804) & 0 & 0 & 0 & 0 & 3 & 0 & 0 & 0 & 0 & 0 & 0 \\
\hline Neottiglossa bifida (A. Costa 1847) & 0 & 0 & 0 & 0 & 0 & 0 & 0 & 0 & 0 & 1 & 0 \\
\hline Palomena prasina (Linnaeus 1761) & 0 & 0 & 0 & 0 & 0 & 0 & 0 & 0 & 0 & 2 & 0 \\
\hline Pentatoma rufipes (Linnaeus 1758) & 0 & 0 & 0 & 1 & 1 & 0 & 0 & 0 & 0 & 1 & 0 \\
\hline Eurygaster maura (Linnaeus 1758) & 0 & 0 & 0 & 0 & 0 & 0 & 0 & 0 & 1 & 0 & 0 \\
\hline Eurygaster testudinaria (Geoffroy 1785) & 0 & 0 & 0 & 0 & 0 & 1 & 0 & 0 & 0 & 2 & 0 \\
\hline Thyreocoris scarabaeoides (Linnaeus 1758) & 0 & 0 & 0 & 0 & 0 & 0 & 2 & 0 & 0 & 2 & 0 \\
\hline Carabus germarii Sturm 1815 & 0 & 0 & 0 & 0 & 0 & 0 & 1 & 0 & 0 & 0 & 0 \\
\hline Agonum sexpunctatum (Linne 1758) & 0 & 0 & 0 & 0 & 2 & 0 & 0 & 0 & 0 & 0 & 0 \\
\hline Platysma oenotrium (Ravizza 1975) & 0 & 2 & 0 & 0 & 0 & 0 & 0 & 0 & 0 & 0 & 0 \\
\hline Pterostichus dissimilis (A. Villa \& G.B. Villa 1833) & 0 & 0 & 0 & 0 & 0 & 0 & 0 & 0 & 3 & 0 & 0 \\
\hline Poecilus versicolor (Sturm 1824) & 0 & 0 & 0 & 0 & 1 & 0 & 0 & 0 & 0 & 0 & 0 \\
\hline Phonias diligens (Sturm 1824) & 0 & 59 & 0 & 3 & 0 & 0 & 0 & 0 & 0 & 0 & 0 \\
\hline Bothriopterus oblongopunctatus (Fabricius 1787) & 1 & 0 & 0 & 0 & 0 & 0 & 0 & 0 & 0 & 0 & 0 \\
\hline Amara eurynota (Panzer 1797) & 0 & 0 & 0 & 0 & 2 & 0 & 0 & 0 & 0 & 0 & 0 \\
\hline Amara lucida (Duftschmid 1812) & 0 & 0 & 0 & 0 & 0 & 0 & 0 & 0 & 1 & 0 & 0 \\
\hline Amara cursitans (Zimmermann 1832) & 0 & 0 & 0 & 0 & 0 & 0 & 0 & 0 & 1 & 0 & 0 \\
\hline Anisodactylus binotatus (Fabricius 1787) & 0 & 0 & 0 & 0 & 1 & 0 & 0 & 0 & 0 & 0 & 0 \\
\hline Acupalpus flavicollis (Sturm 1825) & 0 & 0 & 0 & 0 & 0 & 0 & 0 & 0 & 0 & 1 & 0 \\
\hline Lamprias cyanocephalus (Linne 1758) & 0 & 0 & 0 & 0 & 0 & 0 & 0 & 0 & 2 & 0 & 0 \\
\hline Zeugophora flavicollis (Marsham 1802) & 0 & 0 & 0 & 0 & 1 & 0 & 0 & 0 & 0 & 0 & 0 \\
\hline Donacia obscura Gyllenhal 1813 & 2 & 0 & 0 & 0 & 0 & 0 & 0 & 0 & 0 & 0 & 0 \\
\hline
\end{tabular}




\begin{tabular}{|c|c|c|c|c|c|c|c|c|c|c|c|}
\hline Gonioctena decemnotata (Marsham 1802) & 3 & 2 & 0 & 0 & 1 & 0 & 0 & 0 & 35 & 0 & 0 \\
\hline Gonioctena quinquepunctata (Fabricius 1787) & 4 & 14 & 0 & 0 & 4 & 0 & 0 & 0 & 7 & 6 & 0 \\
\hline Chrysolina fastuosa (Scopoli 1763) & 0 & 0 & 0 & 0 & 0 & 0 & 0 & 0 & 0 & 3 & 0 \\
\hline Chrysolina marginata (Linnaeus 1758) & 0 & 0 & 0 & 0 & 0 & 0 & 0 & 0 & 0 & 3 & 0 \\
\hline Chrysolina geminata (Paykull 1799) & 0 & 0 & 0 & 0 & 0 & 2 & 0 & 0 & 0 & 8 & 0 \\
\hline Chrysomela populi Linnaeus 1758 & 0 & 0 & 0 & 0 & 13 & 0 & 0 & 0 & 0 & 0 & 0 \\
\hline Chrysomela tremulae Fabricius 1787 & 0 & 0 & 0 & 0 & 59 & 0 & 0 & 0 & 0 & 0 & 0 \\
\hline Chrysomela vigintipunctata Scopoli 1763 & 0 & 0 & 0 & 0 & 1 & 0 & 0 & 0 & 0 & 0 & 0 \\
\hline Lochmaea caprea (Linnaeus 1758) & 14 & 6 & 0 & 0 & 0 & 0 & 5 & 0 & 0 & 0 & 0 \\
\hline Galeruca pomonae (Scopoli 1763) & 0 & 0 & 0 & 0 & 1 & 0 & 0 & 0 & 0 & 0 & 0 \\
\hline Galeruca tanaceti (Linnaeus 1758) & 0 & 0 & 0 & 0 & 1 & 0 & 1 & 0 & 0 & 0 & 0 \\
\hline Luperus flavipes (Linnaeus 1767) & 0 & 1 & 0 & 0 & 9 & 0 & 0 & 0 & 1 & 0 & 0 \\
\hline Luperus longicornis (Fabricius 1781) & 0 & 0 & 0 & 0 & 0 & 0 & 0 & 0 & 1 & 0 & 0 \\
\hline Luperus viridipennis Germar 1824 & 0 & 0 & 0 & 0 & 22 & 0 & 1 & 4 & 2 & 0 & 0 \\
\hline Aphthona herbigrada (Curtis 1837) & 0 & 0 & 0 & 0 & 0 & 0 & 0 & 1 & 2 & 0 & 0 \\
\hline Aphthona venustula (Kutschera 1861) & 0 & 0 & 0 & 0 & 6 & 0 & 0 & 1 & 6 & 47 & 0 \\
\hline Longitarsus lewisii (Baly 1874) & 0 & 0 & 0 & 0 & 0 & 0 & 0 & 0 & 1 & 0 & 0 \\
\hline Longitarsus luridus (Scopoli 1763) & 0 & 0 & 0 & 0 & 0 & 0 & 1 & 0 & 0 & 3 & 0 \\
\hline Longitarsus melanocephalus (De Geer 1775) & 0 & 0 & 0 & 0 & 0 & 0 & 0 & 0 & 4 & 0 & 0 \\
\hline Longitarsus pratensis (Panzer 1794) & 0 & 0 & 0 & 0 & 0 & 0 & 0 & 0 & 2 & 0 & 0 \\
\hline Altica oleracea (Linnaeus 1758) & 3 & 6 & 0 & 0 & 8 & 1 & 0 & 0 & 4 & 22 & 0 \\
\hline Bathophila rubi (Paykull 1799) & 0 & 0 & 0 & 0 & 0 & 0 & 0 & 0 & 0 & 1 & 0 \\
\hline Neocrepidodera peirolerii (Kutschera 1860) & 0 & 0 & 0 & 0 & 0 & 0 & 0 & 2 & 0 & 1 & 0 \\
\hline Crepidodera lamina (Bedel 1901) & 0 & 0 & 0 & 0 & 1 & 0 & 0 & 0 & 0 & 0 & 0 \\
\hline Chaetocnema concinna (Marsham 1802) & 0 & 0 & 0 & 0 & 0 & 0 & 0 & 0 & 0 & 2 & 0 \\
\hline Chaetocnema picipes Stephens 1831 & 0 & 0 & 0 & 0 & 0 & 0 & 0 & 0 & 0 & 1 & 0 \\
\hline Chaetocnema hortensis (Geoffroy 1758) & 0 & 0 & 0 & 0 & 0 & 1 & 0 & 0 & 0 & 2 & 0 \\
\hline Chaetocnema sahlbergi (Gyllenhal 1827) & 1 & 2 & 0 & 0 & 0 & 1 & 0 & 0 & 0 & 0 & 0 \\
\hline Smaragdina affinis (Illiger 1794) & 3 & 4 & 0 & 0 & 0 & 0 & 0 & 0 & 7 & 0 & 0 \\
\hline Smaragdina salicina (Scopoli 1763) & 0 & 0 & 0 & 0 & 2 & 1 & 0 & 0 & 0 & 0 & 0 \\
\hline Cryptocephalus elegantulus Gravenhorst 1807 & 1 & 0 & 0 & 0 & 0 & 0 & 0 & 0 & 0 & 0 & 0 \\
\hline Cryptocephalus labiatus Linnaeus 1761 & 1 & 0 & 0 & 0 & 1 & 0 & 2 & 0 & 4 & 0 & 0 \\
\hline Cryptocephalus vittula Suffrian 1848 & 0 & 0 & 0 & 0 & 0 & 0 & 0 & 0 & 0 & 1 & 0 \\
\hline Cryptocephalus bipunctatus (Linnaeus 1758) & 1 & 0 & 0 & 0 & 0 & 0 & 0 & 0 & 0 & 0 & 0 \\
\hline Cryptocephalus flavipes Fabricius 1781 & 0 & 0 & 0 & 0 & 0 & 1 & 0 & 0 & 0 & 0 & 0 \\
\hline Cryptocephalus moraei (Linnaeus 1758) & 0 & 0 & 0 & 0 & 0 & 4 & 0 & 0 & 0 & 0 & 0 \\
\hline Cryptocephalus nitidus (Linnaeus 1758) & 0 & 0 & 0 & 0 & 1 & 0 & 0 & 0 & 1 & 0 & 0 \\
\hline Cryptocephalus quadripustulatus Gyllenhal 1813 & 0 & 0 & 0 & 0 & 0 & 0 & 0 & 0 & 0 & 1 & 0 \\
\hline Cryptocephalus sericeus Marseul 1875 & 0 & 0 & 0 & 0 & 0 & 2 & 0 & 0 & 0 & 0 & 0 \\
\hline Cryptocephalus transiens Franz 1949 & 0 & 0 & 0 & 0 & 0 & 9 & 0 & 0 & 6 & 1 & 0 \\
\hline
\end{tabular}




\begin{tabular}{|l|c|c|c|c|c|c|c|c|c|c|c|}
\hline Bromius obscurus (Linnaeus 1758) & 0 & 0 & 0 & 0 & 6 & 0 & 0 & 0 & 6 & 11 & 0 \\
\hline Cassida sanguinolenta O.F. Müller 1776 & 0 & 0 & 0 & 0 & 0 & 0 & 0 & 0 & 1 & 0 & 0 \\
\hline Otiorhynchus armadillo (Rossi 1792) & 0 & 0 & 0 & 0 & 0 & 0 & 0 & 0 & 0 & 2 & 0 \\
\hline Otiorhynchus scaber (Linnaeus 1758) & 0 & 0 & 0 & 0 & 3 & 0 & 0 & 0 & 0 & 0 & 0 \\
\hline Otiorhynchus frigidus (Mulsant 1859) & 0 & 0 & 0 & 0 & 1 & 0 & 0 & 0 & 0 & 0 & 0 \\
\hline Otiorhynchus anthracinus (Scopoli 1763) & 0 & 0 & 0 & 0 & 0 & 0 & 0 & 0 & 0 & 1 & 0 \\
\hline Phyllobius viridicollis (Fabricius 1792) & 0 & 0 & 0 & 0 & 1 & 0 & 0 & 0 & 0 & 0 & 0 \\
\hline Phyllobius arborator (Herbst 1797) & 1 & 0 & 0 & 0 & 1 & 0 & 0 & 0 & 0 & 0 & 0 \\
\hline Phyllobius pyri (Linnaeus 1758) & 0 & 1 & 0 & 0 & 0 & 0 & 0 & 0 & 1 & 1 & 0 \\
\hline Polydrusus marginatus Stephens 1831 & 0 & 0 & 0 & 0 & 0 & 0 & 0 & 0 & 0 & 1 & 0 \\
\hline Polydrusus cervinus (Linnaeus 1758) & 0 & 0 & 0 & 0 & 0 & 0 & 0 & 1 & 0 & 0 & 0 \\
\hline Sciaphilus asperatus (Bonsdorff 1785) & 0 & 0 & 0 & 0 & 3 & 0 & 0 & 0 & 0 & 1 & 0 \\
\hline Strophosoma melanogrammum (Forster 1771) & 0 & 0 & 0 & 0 & 17 & 0 & 0 & 0 & 0 & 0 & 0 \\
\hline Sitona nigriclavis Stephens 1829 & 0 & 0 & 0 & 0 & 0 & 0 & 1 & 0 & 1 & 0 & 0 \\
\hline SItona sulcifrons (Gyllenhal 1834) & 0 & 0 & 0 & 0 & 2 & 0 & 9 & 0 & 37 & 8 & 0 \\
\hline Lepyrus capucinus (Schaller 1783) & 0 & 0 & 0 & 0 & 0 & 0 & 0 & 0 & 0 & 1 & 0 \\
\hline Hylobius abietis (Linnaeus 1758) & 0 & 0 & 0 & 0 & 0 & 0 & 0 & 0 & 1 & 0 & 0 \\
\hline Magdalis memnonia (Gyllenhal 1832) & 0 & 0 & 0 & 0 & 1 & 0 & 0 & 0 & 1 & 0 & 0 \\
\hline Ceutorhynchus erysimi (Fabricius 1787) & 0 & 0 & 0 & 0 & 1 & 0 & 0 & 0 & 2 & 1 & 0 \\
\hline Nedyus quadrimaculatus (Linnaeus 1758) & 0 & 0 & 0 & 0 & 0 & 0 & 0 & 0 & 0 & 1 & 0 \\
\hline Limnobaris dolorosa (Goeze 1777) & 0 & 1 & 0 & 0 & 0 & 0 & 0 & 0 & 0 & 0 & 0 \\
\hline Limnobaris t-album (Linnaeus 1758) & 0 & 0 & 0 & 0 & 0 & 0 & 0 & 0 & 0 & 2 & 0 \\
\hline Anthonomus rubi (Herbst 1795) & 0 & 0 & 0 & 0 & 0 & 0 & 4 & 0 & 0 & 0 & 0 \\
\hline Tachyerges salicis (Linnaeus 1758) & 0 & 0 & 0 & 0 & 0 & 0 & 0 & 0 & 3 & 1 & 0 \\
\hline Tachyerges stigma (Germar 1821) & 0 & 0 & 0 & 0 & 0 & 0 & 0 & 0 & 0 & 1 & 0 \\
\hline Miarus campanulae Linnaeus 1767 & 0 & 0 & 0 & 0 & 1 & 0 & 0 & 0 & 2 & 3 & 0 \\
\hline
\end{tabular}

species) and 5 (19 species). The zone specificity could be explained by the presence of particular host plants and abiotic ecological requirements that are present in a particular zone. It is worth noting that there were no species specific to zone 4. All the other zones, however, had a number of specific species ranging from 1 (zone 3,8 ) to 18 (zone 5). In general, there were more exclusive species in the surroundings (zones 5, 9, 10) than in the bog area (zones 1, 2, 3,8), with the exception of zone 1 which had 7 exclusive species. From the systematic point of view, both Heteroptera and Coleoptera recorded in this study are characterized by exclusive species, but the highest number of exclusive species belongs to the Heteroptera suborder (27 species) and to the Chrysomelidae family ( 24 species). In zone 6, we found exclusively Chrysomelidae, while the 3 species found in zone 2 belong to three different taxa. Some of the species listed in table 2 , such as the aforementioned $O$. scaber and $O$. frigidus, have a restricted geographical distribution, while others such as Anthonomus rubi (Osella et al., 2005) and Longitarsus pratensis are common species with a wide geographical distribution (Biondi, 2005). 
Table 3 - Species exclusively found in one component of the "Pian di Gembro" wetland (Lombardy, Italy): black squares show species exclusively found in the bog; grey squares show species exclusively found in the surroundings (H: Heteroptera; $C$ : Carabidae; Ch: Chrysomelidae; $\mathrm{Cu}$ : Curculionidae sensu lato; Z1: sampling zone 1; Z2: sampling zone 2; Z3: sampling zone 3; Z4: sampling zone 4; Z5: sampling zone 5; Z6: sampling zone 6; Z7: sampling zone 7; Z8: sampling zone 8; Z9: sampling zone 9; Z10: sampling zone 10; Z11: sampling zone 11).

\begin{tabular}{|c|c|c|c|c|c|c|c|c|c|c|c|c|c|c|c|c|c|c|c|c|c|c|c|c|c|}
\hline & \multicolumn{3}{|c|}{$\mathbf{H}$} & $\mathrm{C}$ & \multicolumn{3}{|c|}{ Ch } & H & $\begin{array}{ll} \\
\end{array}$ & $\mathrm{Cu}$ & $\mathrm{H}$ & \multicolumn{5}{|c|}{$\mathbf{H}$} & \multicolumn{3}{|c|}{$\bar{C}$} & \multicolumn{6}{|c|}{ Ch } \\
\hline 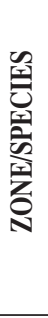 & 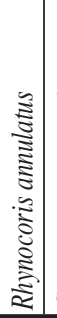 & 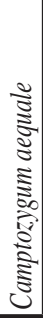 & 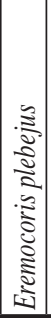 & 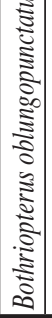 & 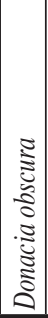 & 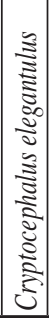 & 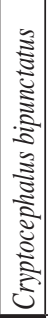 & 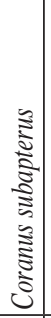 & 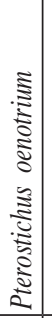 & 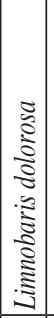 & 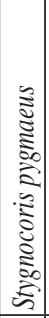 & 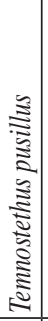 & 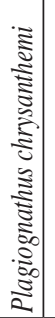 & 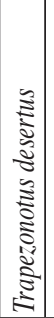 & 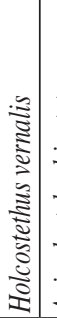 & 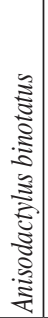 & 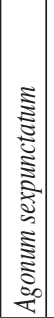 & 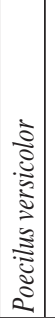 & 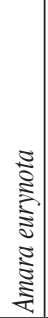 & 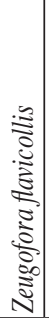 & 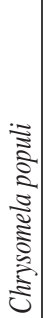 & 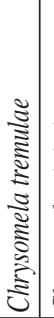 & 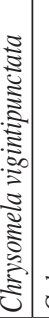 & 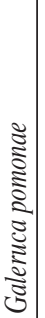 & 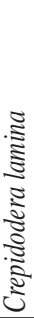 \\
\hline$\overline{\mathrm{Z} 1}$ & & & & & & & & & & & & & & & & & & & & & & & & & \\
\hline $\mathrm{Z2}$ & & & & & & & & & & & & & & & & & & & & & & & & & \\
\hline $\mathrm{Z3}$ & & & & & & & & & & & & & & & & & & & & & & & & & \\
\hline $\mathrm{Z5}$ & & & & & & & & & & & & & & & & & & & & & & & & & \\
\hline & & C & $\mathbf{u}$ & & & $\mathrm{Ch}$ & & & & $\mathrm{H}$ & & & $\begin{array}{ll}\mathrm{C} \\
\end{array}$ & $\mathrm{Cu}$ & $\mathrm{H}$ & I & & & 0 & & & & Ch & & \\
\hline 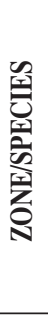 & 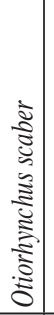 & 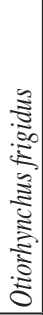 & 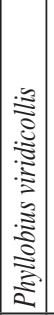 & 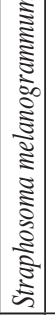 & 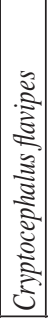 & 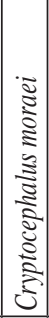 & 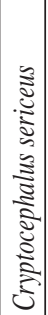 & 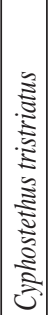 & 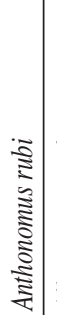 & 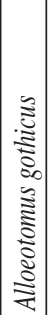 & 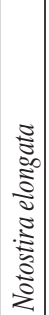 & 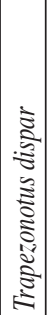 & 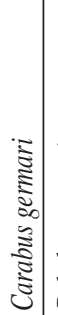 & 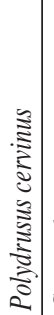 & 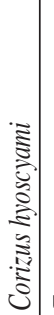 & 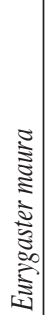 & 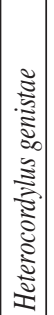 & 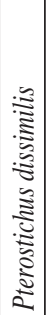 & 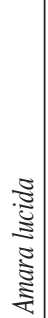 & 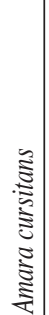 & 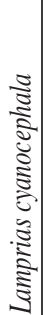 & 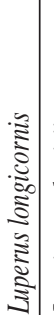 & 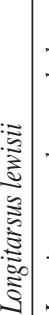 & 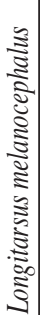 & \\
\hline $\mathbf{Z 5}$ & & & & & & & & & & & & & & & & & & & & & & & & & \\
\hline Z6 & & & & & & & & & & & & & & & & & & & & & & & & & \\
\hline Z7 & & & & & & & & & & & & & & & & & & & & & & & & & \\
\hline Z8 & & & & & & & & & & & & & & & & & & & & & & & & & \\
\hline Z9 & & & & & & & & & & & & & & & & & & & & & & & & & \\
\hline & $\mathrm{Ch}$ & $\mathbf{C u}$ & & & & I & I & & & & C & & & & Ch & & & & & & & $\mathrm{Cu}$ & & & \\
\hline 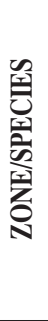 & 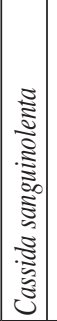 & 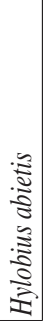 & 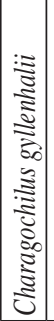 & 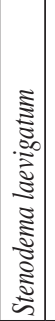 & 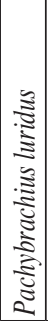 & 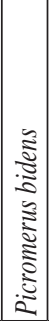 & 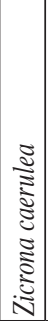 & 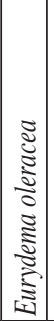 & 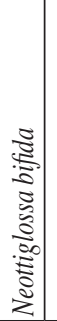 & 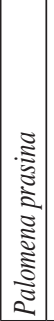 & 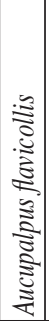 & 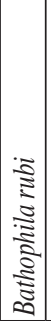 & 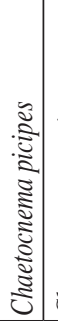 & 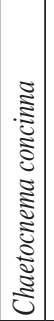 & 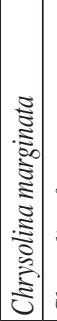 & 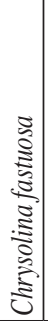 & 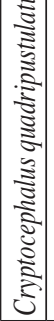 & 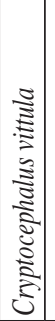 & 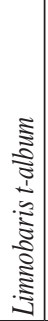 & 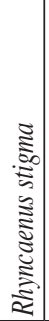 & 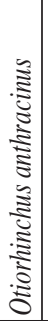 & 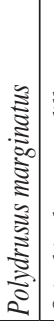 & 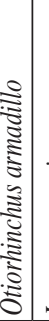 & 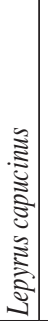 & 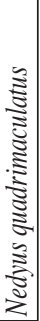 \\
\hline Z9 & & & & & & & & & & & & & & & & & & & & & & & & & \\
\hline & & & & & & & & & & & & & & & & & & & & & & & & & \\
\hline
\end{tabular}


Table 4 - Species found in at least two zones of Pian di Gembro wetland (Lombardy, Italy). Grey squares show species widespread across surrounding zones (group A); black squares show species widespread across both the surrounding and the bog zones (group B) (Z1: sampling zone 1; Z2: sampling zone 2; Z3: sampling zone 3; Z4: sampling zone 4; Z5: sampling zone 5; Z6: sampling zone 6; Z7: sampling zone 7; Z8: sampling zone 8; Z9: sampling zone 9; Z10: sampling zone 10; Z11: sampling zone 11).

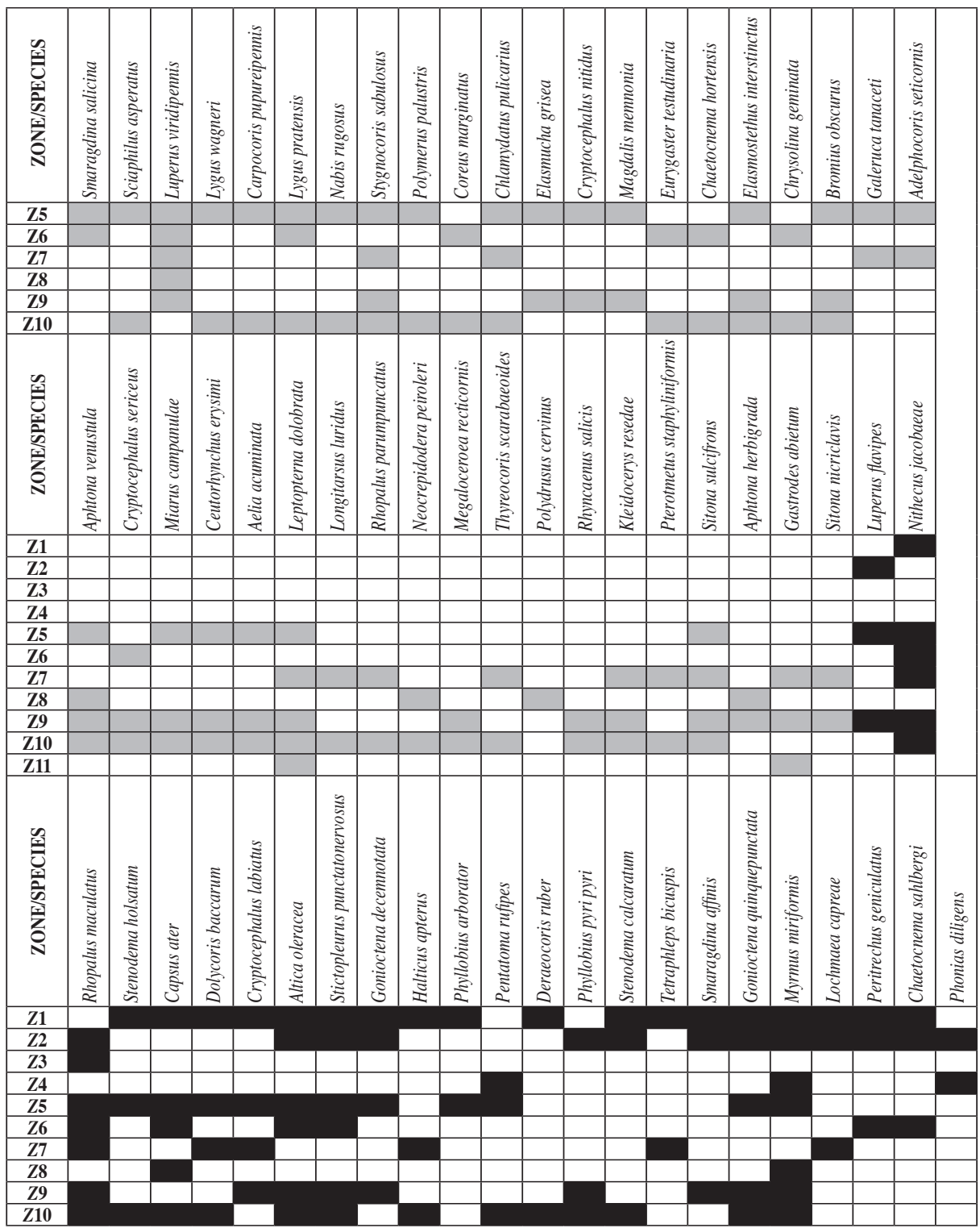


The 64 species ( $45 \%$ of species pool) that occur in more than one zone could be considered as "generalists", with a wider ecological tolerance than species found in one zone only (Table 3 and 4). We split these species into two groups: group A contains "generalist" species widespread in surrounding zones, while group B contains "generalist" species widespread in both surrounding and bog zones. As expected, the outflow zone 8 is inhabited by species occurring in the bog and in the surrounding zones. In group $\mathrm{A}$, there are species of conservation interest and species with a wide geographical distribution such as Lygus pratensis and Galeruca tanaceti, which has also been found in nearby commercial yarrow fields (Limonta et al., 2003; Sassi, 2007) where Penata Gama et al. (2010) studied the dynamics of aphid populations. Within group B, there are species of conservation interest and species with a wide geographical distribution, such as Gonioctena decemnotata and Altica oleracea (Sassi, 2007; Montagna, 2009).

\section{Species of particular conservation interest}

We found 39 species (28\% of the species pool) of conservation interest (Table 5). Pachybrachius luridus is the only species that could be considered a obligate bog or tyrphobiont species linked to Sphagnum spp. and other bog plants (e.g. Carex spp., Rhynchospora sp., Trichophorum sp. and Eriophorum sp.) (Montagna et al., 2008). There were 23 species considered tyrphophiles, including Donacia obscura and Chaetocnema sahlbergi that prefer humid biotopes or microenvironments with high humidity (Doguet, 1994; Montagna, 2009). Five species, including D. obscura and Pterostichus dissimilis, are considered bioindicators of natural environments (Giaccalone et al., 2002; Casale et al., 2005; Sassi, 2005; Montagna et al., 2008; Montagna, 2009). Othiorrynchus scaber, $O$. frigidus, C. sericerus sp. zambanellus and P. dissimilis (2.8\% of species pool) are taxa endemic to the Italian alpine region (Casale et al., 2005; Osella et al., 2005; Sassi, 2005). Three species of Heteroptera (2.1\% of species pool), including Trapezonotus desertus, show a boreo-alpine distribution (Pericart, 1987; Pericart, 1998a, 1998b, 1998c). Nine species of the 'Pian di Gembro' species pool are typical of mountainous regions (e.g. C. sericeus and Amara cursitans are typical alpine taxa), and three are typical of lowland environments (Magistretti, 1965; Biondi, 2005; Casale et al., 2005; Cianficconi, 2005; Sassi, 2005; Montagna et al., 2008). Four species exhibit a wide ecological tolerance (Casale et al., 2005; Montagna et al., 2008). It should also be noted that even at the altitude of $1350 \mathrm{~m}$ above sea level and in the heart of Alps, $2 \%$ of the species are lowland species.

Among the 39 species of conservation interest, there are 25 species that occur in one zone only, 8 in two zones and 6 in more than 2 zones (Table 5).

\section{DISCUSSION}

The insect species pool of 'Pian di Gembro' wetland consists of 141 species collected during three years of sampling. The evaluation of the species pool is difficult because studies on alpine wetland insects are rare and usually restricted to particular taxa. Boyse (2004) studied the insect fauna of acid mires in England and found only 24 species 
Table 5 - Species of Pian di Gembro wetland (Lombardy, Italy) that are considered of conservation interest.

\begin{tabular}{|c|c|c|c|c|c|c|c|c|c|c|c|c|}
\hline SPECIES & งิ & 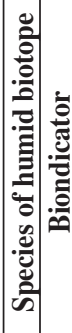 & 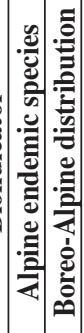 & 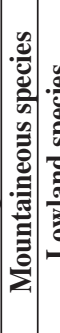 & 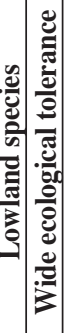 & SPECIES & छัँ & 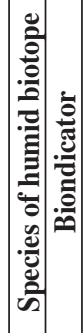 & 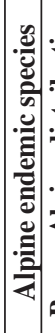 & 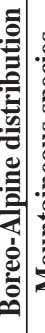 & & 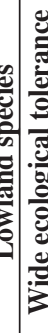 \\
\hline Donacia obscura & 1 & & & & & Limnobaris t-album & 10 & & & & & \\
\hline Rhynocoris annulatus & 1 & & & & & $\begin{array}{l}\text { Cryptocephalus } \\
\text { quadripustulatus }\end{array}$ & 10 & & & & & \\
\hline Coranus subapterus & 2 & & & & & Rhyncaenus stigma & 10 & & & & & \\
\hline Platysma oenotrium & 2 & & & & & Lepyrus capucinus & 10 & & & & & \\
\hline Limnobaris dolorosa & 2 & & & & & $\begin{array}{l}\text { Nabis } \\
\text { flavomarginatus }\end{array}$ & 11 & & & & & \\
\hline Trapezonotus desertus & 5 & & & & & Phonias diligens & 2,4 & & & & & \\
\hline Agonum sexpunctatum & 5 & & & & & $\begin{array}{l}\text { Neocrepidodera } \\
\text { peiroleri }\end{array}$ & 8,9 & & & & & \\
\hline Anisodactylus binotatus & 5 & & & & & \begin{tabular}{|l|} 
Sciaphilus \\
asperatus
\end{tabular} & 5,10 & & & & & \\
\hline $\begin{array}{l}\text { Chrysomela } \\
\text { vigintipunctata }\end{array}$ & 5 & & & & & Polymerus palustris & 5,10 & & & & & \\
\hline Crepidodera lamina & 5 & & & & & $\begin{array}{l}\text { Eurygaster } \\
\text { testudinaria }\end{array}$ & 6,10 & & & & & \\
\hline Otiorhynchus scaber & 5 & & & & & \begin{tabular}{|l|} 
Rhopalus \\
parumpuncatus
\end{tabular} & 7,10 & & & & & \\
\hline Otiorhinchus frigidus & 5 & & & & & $\begin{array}{l}\text { Megaloceroea } \\
\text { recticornis }\end{array}$ & 9,10 & & & & & \\
\hline $\begin{array}{l}\text { Cryptocephalus sericeus } \\
\text { ssp.Zambanellus }\end{array}$ & 6 & & & & & Rhyncaenus salicis & 9,10 & & & & & \\
\hline Anthonomus rubi & 7 & & & & & $\begin{array}{l}\text { Chaetocnema } \\
\text { sahlbergi }\end{array}$ & $1,2,6$ & & & & & \\
\hline Pterostichus dissimilis & 9 & & & & & $\begin{array}{l}\text { Stenodema } \\
\text { calcaratum }\end{array}$ & $1,2,10$ & & & & & \\
\hline Amara cursitans & 9 & & & & & Phyllobius pyri pyri & $2,9,10$ & & & & & \\
\hline Longitarsus lewisii & 9 & & & & & \begin{tabular}{|l} 
Sitona sulcifrons \\
argutulus
\end{tabular} & $\begin{array}{c}5,7,9 \\
10\end{array}$ & & & & & \\
\hline Pachybrachius luridus & 10 & & & & & Nithecus jacobaeae & $\begin{array}{l}1,5,6 \\
7,9,10 \\
\end{array}$ & & & & & \\
\hline Picromerus bidens & 10 & & & & & Rhopalus maculatus & $\begin{array}{c}2,3,5, \\
6,7,9, \\
10\end{array}$ & & & & & \\
\hline Aucupalpus flavicollis & 10 & & & & & & & & & & & \\
\hline
\end{tabular}


belonging to Coleoptera (Carabidae, Chrysomelidae sensu latu, Curculionidae sensu latu) and Heteroptera. Rampazzi \& Dethier (1997) found 105 species of Heteroptera in a bog located in southern Switzerland, and in the Zehlau Bog in the western Russia entomologists collected 38 species of Coleoptera (Carabidae) (Främbs et al., 2002). The results confirm the important contribution of Coleoptera and Heteroptera to the wetland inhabiting insect fauna. Moreover, the studies indicate that the results depend on sampling techniques and sampling efforts. This confirms the importance of building the sampling program on different techniques and justifies their implementation over an extended period of three years.

The collected species grouped into three main coenoses show that 64 species share both wetland components. Nevertheless, the 11 species restricted to the bog and the 63 species found in the surroundings show that a clear difference should be made between the bog and the surroundings. We found 23 tyrphophile insects, which exhibit preferences for boggy areas, but the only obligate bog (tyrphobiont) species found was Pachybrachius luridus. With the exception of zone 4 , all zones are inhabited by zone-specific species (Table 1). Hence, all zones except zone 4 are important in efforts aiming at conserving the general pool of Coleoptera and Heteroptera species. From the standpoint of conserving species of particular interest (Table 5), 24 species are found in at least one out of 8 zones. In the remaining zones, zone 4 and zone 8 share a single species with zone 2 and zone 9 , respectively, while only zone 3 shares the same species with 7 other zones. Hence, the zones 3, 4, 8 could be considered as redundant in efforts to conserve the pool of species of particular interest. By taking into account both the general species pool (Table 1) and the pool of species of particular interest to conservationists (Table 2), only zone 4 can be considered as redundant since it is inhabited by species that occur also in other zones.

In conclusion, with one exception, all the zones are important for conserving the general species pool and the pool of particular interest in conservation programs. As previously described, these zones result from the application of a DIMR aiming at wetland plant species conservation. From the standpoint of conserving the insect species pool, the existing DIMR is also effective for Coleoptera and Heteroptera.

\section{ACKNOWLEDGEMENTS}

We are grateful to Dr. Paride Dioli, Dr. Maurizio Pavesi, Renato Regalin, Dr. Davide Sassi for species identification.

\section{REFERENCES}

ANDReis C., Rodondi G., 1982 - La torbiera di Pian di Gembro (Prov. di Sondrio). - C.N.R. Progr. Fin. "Promozione della qualità dell'ambiente", Roma, AQ/1/221: 1-41.

ANDREIS C., RoDONDI G., 2005 - Tutela della biodiversità della riserva naturale Pian di Gembro in comune di Villa di Tirano. - Piano di Gestione. Rapporto non pubblicato, Università degli Studi di Milano.

Balestrazzi E., Bucciarelli I., Galletti P.A., 1983 - Gli Odonati della torbiera di Pian di Gembro, Trivigno (Sondrio). - Giorn. Ital. Entomol., 1: 211-224. 
Biondi M., 2005 - Insecta Coleoptera Chrysomelidae Alticinae. In: Ruffo S., Stoch F. (ed) Checklist e distribuzione della fauna italiana. - Mem. Mus. Civ. Stor. Nat. Verona, 2 serie, sez. Scienze della Vita, 16: 227-229.

Boyse D.C., 2004 - A review of the invertebrate assemblage of acid mires. - English Nature Research Reports, 592: 1-110.

Casale A., Vigna Taglianti A., Brandmayr P., Colombetta G., 2005 - Insecta Coleoptera Carabidae (Carabini, Cychrini; Trachini; Abacetini, Stomini, Pterostichini). In: Ruffo S., Stoch F. (ed) Checklist e distribuzione della fauna italiana. - Mem. Mus. Civ. Stor. Nat. Verona, 2 serie, sez. Scienze della Vita, 16: 159-163.

Cianficconi F., 2005 - Insecta Trichoptera. In: Ruffo S., Stoch F. (ed) Checklist e distribuzione della fauna italiana. - Mem. Mus. Civ. Stor. Nat. Verona, 2 serie, sez. Scienze della Vita, 16: 251-253.

Cianficconi F., Corallini C., Todini B., 2005 - Tricotterofauna delle Alpi e Prealpi Centrali. Biogeographia, 26: 447-497.

DogUet S., 1994 - Coléoptères Chrysomelidae. Volume II Alticinae. - Faune de France 80, FFSSN, Paris, $694 \mathrm{pp}$.

Erisman J.W., Vermeulen A., Hensen A., Flechard C., Dämmgen U., Fowler D., Sutton M., GRÜNHAGE L., TuOvinen J.P., 2005 - Monitoring and modelling of biosphere/atmosphere exchange of gases and aerosols in Europe. - Environ. Pollut., 133: 403-413.

Fisher B., Kerry Turner R., Morling P., 2009 - Defining and classifying ecosystem services for decision making. - Ecol. Econ. 68: 643-653.

FoCARILE A., 1957 - Sulla coleotterofauna della torbiera di Valle Scoccia (M. Mottarone, Piemonte). - Atti Soc. ital. Sci. Nat. Milan, 96: 85-97.

Främbs H., Dormann W., MassaKowsKi D., 2002 - Spatial distribution of carabid beetle on Zehlau Bog. - Balt. J. Coleopterol. 2: 7-13.

Giaccalone I., Dioli P., Patocchi N., 2002. - Monitoraggi faunistici alle Bolle di Magadino (Svizzera meridionale): eterotteri acquatici e terrestri (Insecta, Heteroptera: Gerromorpha, Nepomorpha e Geocorisae). - Boll. Soc. Ticinese Sci. Nat., 90: 81-92.

Guo Q., 2003 - Disturbance, life history, and optional management for biodiversity. - Ambio, 32: 428-430.

HANSEN A.J., DeFries R., 2007 - Ecological mechanisms linking protected areas to surrounding land. - Ecol. Appl., 17: 974-988.

KRUPA S.V., 2003 - Effects of atmospheric ammonia (NH3) on terrestrial vegetation: a review. Environ. Pollut., 124: 179-221.

Limonta L., Dioli P., Denti A., 2003 - Heteroptera present in two different plant mixture. - Boll. Zool. agr. Bachic., Ser. II, 35: 55-66.

LiU Y., AXMACHER J.C., Li L., WANG C., Yu Z., 2007 - Ground beetle (Coleoptera: Carabidae) inventories: a comparison of light and pitfall trapping. - B. Entomol. Res., 97(6): 577-83.

Magistretti M., 1965 - Coleoptera Cicindelidae Carabidae. Catalogo topografico. - Fauna d'Italia, volume 8. Calderini, Bologna, $512 \mathrm{pp}$.

MARCUZZI C., 1948 - Note sulla biologia di una "torbiera piana" del Trentino - Archo Oceanogr. Limnol., 5: 155-188.

Mason F., Cerretti P., Tagliapietra A., Speight M.C.D., Zapparoli M., 2002 - Invertebrati di una foresta della Pianura Padana, Bosco della Fontana, Primo contributo. - Conservazione Habitat Invertebrati 1. G. Arcari ed., Mantova, 176 pp.

Montagna M., Dioli P., Regalin R., 2008 - Gli Eterotteri terrestri (Insecta, Heteroptera) della torbiera di Pian Gembro (Sondrio, Lombardia). - Il Naturalista Valtellinese, Atti Mus. Civ. Storia Nat. Morbegno, 19: 83-111.

Montagna M., 2009 - Osservazioni sui Crisomelidi delle riserva naturali "Agoraie di Sopra e Moggetto" (Liguria, Genova) e "Guadine Pradaccio" (Emilia-Romagna, Parma) (Coleoptera, Chrysomelidae). - Boll. Ass. Romana entomol., 64: 163-181. 
Osella G., Biondi S., Di Marco C., Magnano L., Zuppa A.M., 2005 - Insecta Coleoptera Curculionoidea. In: Ruffo S., Stoch F. (ed) Checklist e distribuzione della fauna italiana. Mem. Mus. Civ. Stor. Nat. Verona, 2 serie, sez. Scienze della Vita, 16: 231-234.

Penata Gama Z., Morlacchi A., Giorgi A., Lozzia G.C., Baumgärtner J., 2010 - Towards a better understanding of the dynamics of Aphis spiraecola populations in commercial Alpine yarrow fields. - J. Ent. Acar. Res. Ser. II, 42 (2): 103-116.

PericART J., 1987-Hémiptères Nabidae d'Europe occidentale et du Maghreb.- Faune de France 71, FFSSN, Paris, 186 pp.

PericART J., 1998 - Hémiptéres Lygaeidae euro-méditerranéens, volume 1. - Faune de France 84a, FFSSN, Paris, 468 pp.

PericART J., 1998 - Hémiptéres Lygaeidae euro-méditerranéens, volume 2. - Faune de France 84b, FFSSN, Paris, 453 pp.

Pericart J., 1998 - Hémiptéres Lygaeidae euro-méditerranéens, volume 3. - Faune de France $84 \mathrm{c}$, FFSSN, Paris, $487 \mathrm{pp}$.

Possingham H.P., Wilson K.A., Andelman S.J., Vynne C.H., 2006 - Protected areas: goals, limitations, and design. In: Groom M.J., Meefe G.K., Carroll C.R. (ed) - Principles of Conservation Biology, 3rd ed. Sinauer Associates, Sunderland: 509-533.

RAMPAZZi F., DEthIER M., 1997 - Gli Eterotteri (Insecta: Heteroptera) delle torbiere a sfagni del Cantone Ticino e del Moesano (Val Calanca e Val Mesolcina-GR), Svizzera. - Mitt. Schweiz. Entomol. Ges., 70: 419-439.

SASSI D., 2005 - Insecta Coleoptera Chrysomelidae Cryptocephalinae. In: Ruffo S., Stoch F (ed) Checklist e distribuzione della fauna italiana. - Mem. Mus. Civ. Stor. Nat. Verona, 2 serie, sez. Scienze della Vita, 16: 223-225.

SAssi D., 2007 - I Crisomelidi (Coleoptera Chrysomelidae) del Triangolo Lariano (Italia, Lombardia). Società Italiana di Scienze Naturali e Museo Civico di Storia Naturale di Milano. - Natura, 96: 1-44.

Smith T.L., Sмith R.L., 2001 - Elements of ecology, 6th ed. Pearson Educations, Old Tappan NJ, 736 pp.

SPITZER K., DANKs H.V., 2006 - Insect Biodiversity of Boreal Peat Bogs. - Annu. Rev. Entomol., 51: 137-161.

TURNER R.K., VAN DEN Bergh J.C.J.M., SÖDERQvist T., B ARENDREgT A., VAN DER STRAaten J., MALTBY E., vAN IERLAND E.C., 2000 - Ecological-economic analysis of wetlands: scientific integration for management and policy. - Ecol. Econ., 35: 7-23.

Uys C., Hamer M., Slotow R., 2010 - Step process for selecting and testing surrogates and indicators of afrotemperate forest invertebrate diversity. - PLoS One, 9: 5 (2): e9100.

Matteo Montagna, Dipartimento di Patologia Animale, Igiene e Sanità Pubblica Veterinaria, Università degli Studi di Milano, Milano, Italy; Dipartimento di Protezione dei Sistemi Agroalimentare e Urbano e Valorizzazione delle Biodiversità, Università degli Studi di Milano, Milano, Italy. e-mail: matteo.montagna@unimi.it.

Giuseppe Carlo Lozzia, Johann Baumgärtner, Dipartimento di Protezione dei Sistemi Agroalimentare e Urbano e Valorizzazione delle Biodiversità, Università degli Studi di Milano, Milano.

Carlo Andreis, Dipartimento di Biologia, sezione di Botanica Sistematica e Geobotanica, Università degli Studi di Milano, Milan, Italy.

ANNA GIORGI, GeSDiMont, Centro di studi applicati per la gestione sostenibile della montagna. Università degli Studi di Milano, Edolo, Italy. 